\title{
SEPARATION OF STREAMLINES FOR SPATIALLY PERIODIC FLOW AT ZERO REYNOLDS NUMBERS*
}

BY

\author{
K. B. RANGER
}

University of Toronto, Toronto, Canada

\begin{abstract}
The stream function is found for the creeping flow between two cylinders. The inner cylinder is approximately circular and is rotating with constant angular velocity. The outer cylinder is fixed and corrugated or spatially periodic. The boundary vorticity on the outer boundary is discussed in relation to separation of the streamlines, as a function of the parameters describing the boundary geometry.
\end{abstract}

Introduction. There is engineering interest in connection with flow in the presence of a corrugated or spatially periodic boundary. The present paper describes the creeping two-dimensional motion between two cylinders, the inner of which is approximately circular and the outer of which is fixed and corrugated. The inner cylinder rotates with constant angular velocity $\omega$.

The generalized fluted column transformation [2] is used to describe the boundary geometry and the flow is modelled by constructing suitable spatially periodic solutions of the biharmonic equation appropriate to the boundary conditions. A solution for the stream function is found in a finite form as a function of the mapped coordinates and is correct to $O\left(\lambda^{n}\right)$ where $\lambda<1$ is the radius of the inner boundary and $n$ is the number of peaks of outer boundary corrugation. Alternatively, the expression found for the stream function may be interpreted as the exact solution for a two-dimensional line rotlet located at the center and in the presence of the outer corrugated cylinder.

The vorticity and pressure field are calculated on the outer boundary and, for certain relations satisfied by the parameters describing the geometry of the outer cylinder, there is separation of the streamlines. There are many examples of flow separation of zero Reynolds number [3] but the solution found here seems to be new for a periodic boundary. Simple expressions for the torques are given as functions of the boundary parameters.

The generalized fluted column transformation. This transformation is defined by

$$
z=\zeta+\sum_{s=1}^{N} \varepsilon_{s} \zeta^{s n+1}, \quad z=x+i y=r e^{i \theta}
$$

${ }^{*}$ Received August 10, 1988. 


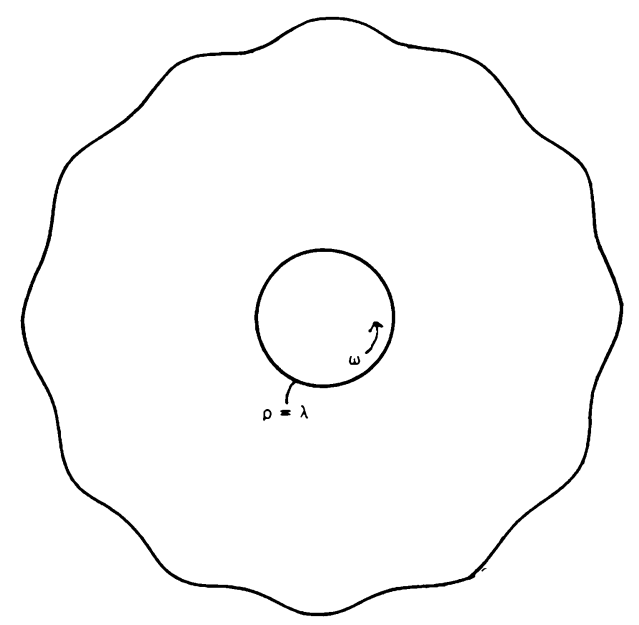

Fig. 1. Sketch of the fluted column with $N=12$.

In this case $\varepsilon_{1}\left(1+\varepsilon_{2}\right)>4 \varepsilon_{2}$ and the inner peaks occur at $\cos n \phi=-1$.

and $\zeta=\rho e^{i \phi}$. The case $N=1$ originates in [1] in connection with the torsion problem in two-dimensional isotropic elasticity. For $N \geq 1$ the transformation is regular analytic in the interior of $|\zeta|=1$ when $n$ is a positive integer and if $0<\varepsilon_{s}<1$, $s=1, \ldots, N$, with $m_{s}=\varepsilon(s n+1)$ then for

$$
1>m_{1}>m_{2}>\cdots>m_{N}>0
$$

the roots of

$$
\frac{d z}{d \zeta}=1+\sum_{s=1}^{N} m_{s} \zeta^{s n}
$$

lie outside the unit circle $|\zeta|=1$ and the transformation is conformal within $|\zeta|=1$. This result depends on the example given in [2]. The case to be considered in the present paper is $N=2$, so that

$$
z=\zeta+\varepsilon_{1} \zeta^{n+1}+\varepsilon_{2} \zeta^{2 n+1}
$$

where $0<\varepsilon_{j}<1, j=1,2$, and $0<m_{2}<m_{1}<1$ with $m_{1}=(n+1) \varepsilon_{1}, m_{2}=(2 n+1) \varepsilon_{2}$. In terms of $x, y$ the mapping can be written as

$$
\begin{aligned}
& x=\rho \cos \phi+\varepsilon_{1} \rho^{n+1} \cos (n+1) \phi+\varepsilon_{2} \rho^{2 n+1} \cos (2 n+1) \phi, \\
& y=\rho \sin \phi+\varepsilon_{1} \rho^{n+1} \sin (n+1) \phi+\varepsilon_{2} \rho^{2 n+1} \sin (2 n+1) \phi,
\end{aligned}
$$

so that

$$
\begin{aligned}
r^{2}=x^{2}+y^{2}= & \rho^{2}+2 \varepsilon_{1} \rho^{n+2} \cos n \phi+2 \varepsilon_{1} \varepsilon_{2} \rho^{3 n+2} \cos n \phi \\
& +2 \varepsilon_{2} \rho^{2 n+2} \cos 2 n \phi+\varepsilon_{1}^{2} \rho^{2 n+2}+\varepsilon_{2}^{2} \rho^{4 n+2}
\end{aligned}
$$

and, in particular on $\rho=1$,

$$
r^{2}=1+2 \varepsilon_{1}\left(1+\varepsilon_{1}+\varepsilon_{2}\right)^{2}-2(1-\cos n \phi)\left[\varepsilon_{1}\left(1+\varepsilon_{2}\right)+2 \varepsilon_{2}(1+\cos n \phi)\right] .
$$

The absolute maxima of $r^{2}$ occur at $\cos n \phi=1$ where there are $n$ peaks and $r=1+\varepsilon_{1}+$ $\varepsilon_{2}$. There are extremal points at $\cos n \phi=-1$ and also at $\cos n \phi=-\varepsilon_{1}\left(1+\varepsilon_{2}\right) /\left(4 \varepsilon_{2}\right)$ if 
$\varepsilon_{1}\left(1+\varepsilon_{2}\right) /\left(4 \varepsilon_{2}\right)<1$. If $\varepsilon_{1}\left(1+\varepsilon_{2}\right) /\left(4 \varepsilon_{2}\right)>1$, then the minimum occurs at $\cos n \phi=-1$, where $r=1-\varepsilon_{1}+\varepsilon_{2}$ but if $\varepsilon_{1}\left(1+\varepsilon_{2}\right) /\left(4 \varepsilon_{2}\right)<1$ then the minimum occurs at $\cos n \phi=-\varepsilon_{1}\left(1+\varepsilon_{2}\right) /\left(4 \varepsilon_{2}\right)<1$ where

$$
r^{2}=\left(r-\varepsilon_{2}\right)^{2}\left[1-\varepsilon_{1}^{2} /\left(r \varepsilon_{2}\right)\right]
$$

and the points $\cos n \phi=-1$ correspond to local maxima. In this latter case there are $2 n$ inner peaks where $r$ is a minimum while if $\varepsilon_{1}\left(1+\varepsilon_{2}\right) /\left(4 \varepsilon_{2}\right)<1$, there are $n$ inner peaks where $r$ is a minimum. Sketches of the fluted column for $\varepsilon_{1}\left(1+\varepsilon_{2}\right) \geq 4 \varepsilon_{2}$ are given in Figs. 2 and 3.

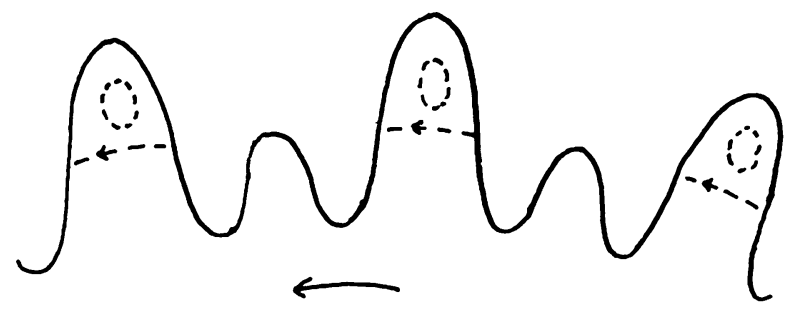

FIG. 2. Sketch of the fluted column for $\varepsilon_{1}\left(1+\varepsilon_{2}\right)<4 \varepsilon_{2}$.

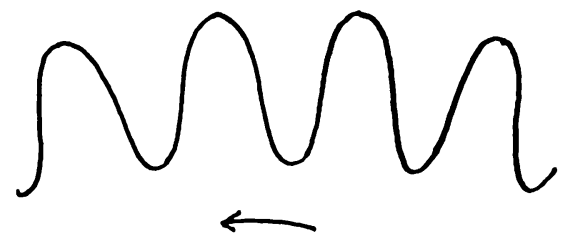

Fig. 3. Sketch of the fluted column for $\varepsilon_{1}\left(1+\varepsilon_{2}\right)>4 \varepsilon_{2}$.

Again if $0<\lambda<1$ with $\lambda^{n} \ll 1$, then on $\rho=\lambda$,

$$
r^{2}=\lambda^{2}+2 \varepsilon_{1} \lambda^{n+2} \cos n \phi+2 \varepsilon_{1} \varepsilon_{2} \lambda^{3 n+2} \cos n \phi+2 \varepsilon_{2} \lambda^{2 n+2} \cos 2 n \phi+\varepsilon_{1}^{2} \lambda^{2 n+2}+\varepsilon_{2}^{2} \lambda^{4 n+2}
$$

so that

$$
r^{2} \approx \lambda^{2}
$$

and the region $\lambda \leq \rho \leq 1$ maps into the region between the approximate circle $r=\lambda$, and the fluted column $\rho=1$. For example if $\lambda=0.5, n=12, \lambda^{n}<2.10^{-4}$.

The flow problem. The flow problem to be considered is the creeping motion between the approximate circular cylinder which is rotating with constant nondimensional angular velocity $\omega$, and the corrugated outer cylinder which is fixed.

The nondimensional fluid velocity $q$ can be described in terms of a stream function $\psi$ by

$$
\mathbf{q}=\operatorname{curl}(-\psi \hat{\mathbf{k}})=-\psi_{y} \hat{\mathbf{i}}+\psi_{x} \hat{\mathbf{j}}
$$

and $\psi$ satisfies the nondimensional Stokes equations

$$
\frac{\partial p}{\partial x}=-\frac{\partial}{\partial y} \nabla^{2} \psi, \quad \frac{\partial p}{\partial y}=\frac{\partial}{\partial x} \nabla^{2} \psi
$$


where $p$ is the pressure field, and $\nabla^{2}$ is the two-dimensional Laplacian defined by

$$
\nabla^{2} \equiv \frac{\partial^{2}}{\partial x^{2}}+\frac{\partial^{2}}{\partial y^{2}}
$$

Elimination of the pressure field from (13) yields the biharmonic equation for $\psi$, viz.,

$$
\nabla^{4} \psi=0
$$

The boundary conditions of zero velocity on the corrugated boundary $\rho=1$ are satisfied if

$$
\psi=\text { constant }, \quad \frac{\partial \psi}{\partial \rho}=0 \quad \text { at } \rho=1 .
$$

The inner boundary conditions are satisfied approximately if

$$
\psi=\text { constant }, \quad \frac{\partial \psi}{\partial \rho}=\omega \lambda \quad \text { at } \rho=\lambda
$$

provided $\lambda^{n} \ll 1$. The boundary conditions force spatially periodic solutions of the biharmonic equation. Using the fact that the two-dimensional Laplace equation is invariant under conformal mapping, suitable solutions can be constructed in the following way:

$$
\begin{aligned}
\psi_{1} & =x \rho \cos \phi+y \rho \sin \phi=\rho^{2}+\varepsilon_{1} \rho^{n+2} \cos n \phi+\varepsilon_{2} \rho^{2 n+2} \cos 2 n \phi, \\
\psi_{2} & =x \rho^{n+1} \cos (n+1) \phi+y \rho^{n+1} \sin (n+1) \phi \\
& =\rho^{n+2} \cos n \phi+\varepsilon_{1} \rho^{2 n+2}+\varepsilon_{2} \rho^{3 n+2} \cos n \phi, \\
\psi_{3} & =x \rho^{2 n+1} \cos (2 n+1) \phi+y \rho^{2 n+1} \sin (2 n+1) \phi \\
& =\rho^{2 n+2} \cos 2 n \phi+\varepsilon_{1} \rho^{3 n+2} \cos n \phi+\varepsilon_{2} \rho^{4 n+2} .
\end{aligned}
$$

A solution appropriate to the present flow is given by

$$
\psi=A \log \rho+B \psi_{2}+C \psi_{4}+D \rho^{n} \cos n \phi,
$$

where

$$
\psi_{4}=\psi-\varepsilon_{2} \psi_{3}=\rho^{2}-\varepsilon_{2}^{2} \rho^{4 n+2}+\left[\varepsilon_{1} \rho^{n+2}-\varepsilon_{1} \varepsilon_{2} \rho^{3 n+2}\right] \cos n \phi .
$$

The stream function $\psi$ may be written as

$$
\begin{aligned}
\psi=A \log \rho & +B\left\{\left[\rho^{n+2}+\varepsilon_{2} \rho^{3 n+2}\right] \cos n \phi+\varepsilon_{1} \rho^{2 n+2}\right\} \\
& +C\left\{\rho^{2}-\varepsilon_{2}^{2} \rho^{4 n+2}+\varepsilon_{1}\left(\rho^{n+2}-\varepsilon_{2} \rho^{3 n+2}\right) \cos n \phi\right\}+D \rho^{n} \cos n \phi
\end{aligned}
$$

where $A, B, C, D$ are constants to be determined by the boundary conditions. After application of these conditions the constants satisfy the system of equations

$$
\begin{gathered}
B\left(1+\varepsilon_{2}\right)+C \varepsilon_{1}\left(1-\varepsilon_{2}\right)+D=0, \\
B\left[n+2+\varepsilon_{2}(3 n+2)\right]+C \varepsilon_{1}\left[n+2-\varepsilon_{2}(3 n+2)\right]+n D=0, \\
A+2 \varepsilon_{1}(n+1) B+2 C\left[1-(2 n+1) \varepsilon_{2}^{2}\right]=0,
\end{gathered}
$$

and neglecting terms of $O\left(\lambda^{n}\right)$ the inner conditions are satisfied if

$$
\omega \lambda^{2}=A+2 C \lambda^{2} \text {. }
$$


Elimination of $A, D$ from the above system yields the equations

$$
\begin{gathered}
\omega \lambda^{2}=2 C\left[\lambda^{2}-1+(2 n+1) \varepsilon_{2}^{2}\right]-2(n+1) \varepsilon_{1} B, \\
B\left[1+(n+1) \varepsilon_{2}\right]+C \varepsilon_{1}\left[1-(n+1) \varepsilon_{2}\right]=0 .
\end{gathered}
$$

The constants $B$ and $C$ are then found to be

$$
\begin{aligned}
& B=\frac{-\frac{1}{2} \omega \lambda^{2} \varepsilon_{1}\left[1-(n+1) \varepsilon_{2}\right]}{\left\{\left[\lambda^{2}-1+(2 n+1) \varepsilon_{2}^{2}\right]\left[1+(n+1) \varepsilon_{2}\right]+(n+1) \varepsilon_{1}^{2}\left[1-(n+1) \varepsilon_{2}\right]\right\}}, \\
& C=\frac{\frac{1}{2} \omega \lambda^{2} \varepsilon_{1}\left[1+(n+1) \varepsilon_{2}\right]}{\left\{\left[\lambda^{2}-1+(2 n+1) \varepsilon_{2}^{2}\right]\left[1+(n+1) \varepsilon_{2}\right]+(n+1) \varepsilon_{1}^{2}\left[1-(n+1) \varepsilon_{2}\right]\right\}},
\end{aligned}
$$

and the stream function $\psi$ is expressed by

$$
\begin{aligned}
\psi= & \left(\omega \lambda^{2}-2 C \lambda^{2}\right) \log \rho+B\left\{\left(\varepsilon_{2} \rho^{3 n+2}+\rho^{n+2}\right) \cos n \phi+\varepsilon_{1} \rho^{3 n+2}\right\} \\
& +C\left\{\rho^{2}-\varepsilon_{2}^{2} \rho^{4 n+2}+\varepsilon_{1}\left(\rho^{n+2}-\varepsilon_{2} \rho^{3 n+2}\right) \cos n \phi\right\} \\
& -\left\{B\left(1+\varepsilon_{2}\right)+C \varepsilon_{1}\left(1-\varepsilon_{2}\right)\right\} \rho^{n} \cos n \phi .
\end{aligned}
$$

As a check on the stream function in the case $\varepsilon_{1}=\varepsilon_{2}=0$, Eq. (32) reduces to

$$
\psi=\frac{\omega \lambda^{2}}{1-\lambda^{2}} \log \rho-\frac{1}{2} \frac{\omega \lambda^{2}}{1-\lambda^{2}} \rho^{2},
$$

which is the known stream function for flow between concentric circles in which the inner $\rho=\lambda$ rotates with angular velocity $\omega$ and the outer $\rho=1$ is fixed. In the case $\varepsilon_{2}=0$, the stream function is a function of $\rho$ only and is given by

$$
\psi=\frac{\omega \lambda^{2}}{\left[1-\lambda^{2}-(n+1) \varepsilon_{1}^{2}\right]}\left\{\left[1-(n+1) \varepsilon_{1}^{2}\right] \log \rho-\frac{1}{2}\left[\rho^{2}-\varepsilon_{1}^{2} \rho^{2 n+2}\right]\right\} ;
$$

so the streamlines coincide with the fluted columns $\rho=$ constant, corresponding to $N=1$. Clearly there is no separation of the streamlines at the corrugated boundary in this case.

The vorticity $\zeta_{1}$ is defined by

$$
\zeta_{1}=\nabla^{2} \psi=\frac{W}{|d z / d \zeta|^{2}}
$$

where

$$
W=\psi_{\rho \rho}+\frac{1}{\rho} \psi_{\rho}+\frac{1}{\rho^{2}} \psi_{\phi \phi}
$$

and

$\left|\frac{d z}{d \zeta}\right|^{2}=1+m_{1}^{2} \rho^{2 n}+m_{2}^{2} \rho^{4 n}+2 m_{2} \rho^{2 n} \cos 2 n \phi+2 m_{1} \rho^{n} \cos n \phi+2 m_{1} m_{2} \rho^{3 n} \cos n \phi$.

A straightforward calculation shows that

$$
\begin{aligned}
W= & 4 B(n+1)\left\{\left[\rho^{n}+(2 n+1) \varepsilon_{2} \rho^{3 n}\right] \cos n \phi+\varepsilon_{1}(n+1) \rho^{2 n}\right\} \\
+ & 4 C\left\{1-(2 n+1)^{2} \varepsilon_{2}^{2} \rho^{4 n}+(n+1) \varepsilon_{1} \rho^{n} \cos n \phi\right. \\
& \left.-(2 n+1)(n+1) \rho^{3 n} \varepsilon_{1} \varepsilon_{2} \cos n \phi\right\}
\end{aligned}
$$


so that the vorticity is given by

$$
\begin{aligned}
\zeta_{1}=\nabla^{2} \psi=\left[4 B(n+1)\left\{\left[\rho^{n}+(2 n+1) \varepsilon_{2} \rho^{3 n}\right] \cos n \phi+\varepsilon_{1}(n+1) \rho^{2 n}\right\}\right. \\
+4 C\left\{1-(2 n+1)^{2} \varepsilon_{2}^{2} \rho^{4 n}+(n+1) \varepsilon_{1} \rho^{n} \cos n \phi\right. \\
\left.\left.-(2 n+1)(n+1) \varepsilon_{1} \varepsilon_{2} \rho^{3 n} \cos n \phi\right\}\right] \\
\times\left[1+m_{1}^{2} \rho^{2 n}+m_{2}^{2} \rho^{4 n}+2 m_{2} \rho^{2 n} \cos 2 n \phi\right. \\
\left.+2 m_{1} \rho^{n} \cos n \phi+2 m_{1} m_{2} \rho^{3 n} \cos n \phi\right]^{-1} .
\end{aligned}
$$

From Eq. (13), $\zeta_{1}$ and $p$ are conjugate two-dimensional harmonics and the pressure field is expressed by

$$
\begin{aligned}
p= & 4(n+1) B\left[\rho^{n} \sin n \phi-m_{2} \rho^{3 n} \sin n \phi\right] \\
& +4 C\left[-2 m_{2} \rho^{2 n} \sin 2 n \phi-m_{1} m_{2} \rho^{3 n} \sin n \phi-m_{1} \rho^{n} \sin n \phi\right] \\
& \times\left[1+m_{1}^{2} \rho^{2 n}+m_{2}^{2} \rho^{4 n}+2 m_{2} \rho^{2 n} \cos 2 n \phi+2 m_{1} \rho^{n} \cos n \phi+2 m_{1} m_{2} \rho^{3 n} \cos n \phi\right]^{-1}
\end{aligned}
$$

On the corrugated boundary $\rho=1$,

$$
W(1, \phi)=-4 C m_{1} \gamma\left[\left(1+m_{2}\right) \cos n \phi+m_{1}\right]+4 C\left[1-m_{2}^{2}+m_{1}\left(1-m_{2}\right) \cos n \phi\right]
$$

where

$$
\gamma=\frac{1-(n+1) \varepsilon_{2}}{1+(n+1) \varepsilon_{2}}
$$

In particular at $\cos n \phi=1$,

$$
W=4 C\left[1-m_{2}-m_{1} \gamma\right]\left(1+m_{1}+m_{2}\right)
$$

and at $\cos n \phi=-1$,

$$
W=4 C\left[1-m_{2}+\gamma m_{1}\right]\left(1+m_{2}-m_{1}\right) .
$$

Since $C<0$, and $\gamma>0,0<m_{2}<m_{1}<1$, the vorticity at $\cos n \phi=-1$ is negative and there is no separation of streamlines at these points. Separation occurs only when the boundary vorticity is positive and this will take place at points where $\cos n \phi=1$ which corresponds to the maximum distance of the corrugated boundary from the origin, provided that

$$
1-m_{2}-m_{1} \gamma<0
$$

For example, if $m_{1}=\frac{7}{8}, m_{2}=\frac{3}{4}$,

$$
1-m_{2}-m_{1} \gamma=\frac{\left(9 \varepsilon_{2}-13\right)}{8\left(11+\varepsilon_{2}\right)}<0
$$

since $0<\varepsilon_{2}<1$. Again since $\cos n \phi \leq 1$ the separation first occurs at the points $\cos n \phi=1$ considered as a function of the boundary parameters and spreads around the boundary but not reaching points where $\cos n \phi=-1$. In fact, for this particular choice of $m_{1}$ and $m_{2}, 4 \varepsilon_{2}>\varepsilon_{1}\left(1+\varepsilon_{2}\right)$ and the minimum values of $r$ occur at $\cos n \phi=$ $-\varepsilon_{1}\left(1+\varepsilon_{2}\right) /\left(4 \varepsilon_{2}\right)$ from which it is readily shown that the vorticity is negative at these peaks. It follows that the separated region of flow is confined to the most concave regions of the boundary in agreement with the results of Stokes flow separation [3]. There is no separated flow in the regions $-\varepsilon_{1}\left(1+\varepsilon_{2}\right) /\left(4 \varepsilon_{2}\right) \geq \cos n \phi \geq-1$. As already 
pointed out there is no separation of the flow for $m_{2}=0$. See Fig. 1. However, the boundary vorticity is expressed by

$$
\zeta_{1}=\left.\nabla^{2} \psi\right|_{\rho=1}=\frac{-2 \omega \lambda^{2}\left(1-m_{1}^{2}\right)}{\left[1-\lambda^{2}-m_{1} \varepsilon_{1}\right]\left[1+2 m_{1} \cos n \phi+m_{1}^{2}\right]}
$$

and if $m_{1}=1-\delta, 0<\delta \ll 1$, so that $\zeta_{1}=O(\delta)$ at $\cos n \phi=1$ and $O\left(\delta^{-1}\right)$ at $\cos n \phi=-1$.

The torques. Neglecting terms of $O\left(\lambda^{n}\right)$ the torque per unit thickness of inner cylinder is found from the standard formula as

$$
\begin{aligned}
G & =2 \pi\left\{2 C \lambda^{2}-\omega \lambda^{2}\right\} \\
& =2 \pi \lambda \omega\left\{\frac{\lambda^{2}\left[1+(n+1) \varepsilon_{2}\right]}{\left[\lambda^{2}-1+(2 n+1) \varepsilon_{2}^{2}\right]\left[1+(n+1) \varepsilon_{2}\right]+(n+1) \varepsilon_{1}^{2}\left[1-(n+1) \varepsilon_{2}\right]}-1\right\} .
\end{aligned}
$$

The torque on the outer cylinder is $-G$. It is perhaps of interest to point out that even in the case of separated flow the corrugated boundary has little influence on the torque in comparison to that of circular cylinders, the difference being of $O\left(n^{-1}\right)$.

\section{REFERENCES}

[1] D. E. R. Godfrey, Theoretical Elasticity and Plasticity for Engineers, London: Thames and Hudson, 1959, pp. 58-59

[2] E. G. Phillips, Functions of a Complex Variable with Applications, Oliver and Boyd Interscience Publishers, Inc., 1958, p. 31

[3] M. E. O'Neill and K. B. Ranger, Handbook of Multiphase Systems, Ed. G. Hetsroni, Hemisphere Publishing Corporation, 1982, pp. 96-204 